FERMILAB-TM-1876

\title{
The Future of High Energy Physics in the United States Statement for the Science Subcommittee of the House Science, Space and Technology Committee
}

\author{
John Peoples, Jr. \\ Fermi National Accelerator Laboratory \\ P.O. Box 500, Batavia, Illinois 60510
}

January 1994 


\section{Disclaimer}

This report was prepared as an account of work sponsored by an agency of the United States Government. Neither the United States Government nor any agency thereof, nor any of their employees, makes any warranty, express or implied, or assumes any legal liability or responsibility for the accuracy, completeness, or usefulness of any information, apparatus, product, or process disclosed, or represents that its use would not infringe privately owned rights. Reference herein to any specific commercial product, process, or service by trade name, trademark, manufacturer, or otherwise, does not necessarily constitute or imply its endorsement, recommendation, or favoring by the United States Government or any agency thereof. The views and opinions of authors expressed herein do not necessarily state or reflect those of the United States Government or any agency thereof. 


\title{
The Future of High-Energy Physics in the United States
}

\author{
Statement for the Science Subcommittee of the \\ House Science, Space, and Technology Committee \\ John Peoples Jr. \\ Fermi National Accelerator Laboratory \\ January 26, 1994
}

\section{The Capability for Excellence}

The United States has a superb capability for forefront research in elementary particle physics for the next decade-but it can be realized only if there is sufficient funding to make efficient use of our existing world-class facilities. Research in particle physics over the past sixty years has produced a remarkably successful theoretical picture describing matter and energy as built of certain constituents, interacting through specific forces according to known physical laws. Yet despite its success, this picture also raises profound questions that only new experiments can answer. These questions present great opportunities for discovery. Throughout most of the long period of its development, the U.S. led the world in making contributions to the understanding of matter and energy. Today the U.S. is still one of the leaders in this global field of research. In spite of the termination of the SSC, the U.S. can make many important contributions to elementary particle physics for the remainder of this decade. But, after that, we will not remain among the leaders in the ensuing decades unless we rebuild our capability to construct and operate world-class facilities.

\section{The Structure of Research}

United States high-energy physics experimentalists are primarily university faculty and students who carry out their research at high-energy laboratories throughout the world. The four U.S. high-energy laboratories that provide most of their research opportunities are: the Tevatron at Fermilab, the Alternating Gradient Synchrotron (AGS) at Brookhaven National Laboratory, the electron linac and storage rings at the Stanford Linear Accelerator (SLAC), and the Cornell Electron Storage Ring (CESR). Funds for the operation of the first three are provided by DOE, while NSF funds the operation of CESR. Besides particle accelerators, these laboratories provide the resources for construction of the detectors that track the results of particle collisions; and they provide the computing to manage and analyze the extraordinary amount of data acquired with these detectors. The detectors, major facilities in themselves, are conceived and designed by the experimental collaborations; detector construction is managed by the laboratory and the collaboration. It takes as much time to build a major detector as it does to build a collider-typically 10 years. Thus, building a detector demands a long-term commitment, not only from the laboratory but also from the universities who will collaborate in building and operating it. 
This long time scale means that stable funding is critical for success in the field.

The university experimentalists in high-energy physics are supported by DOE and NSF, and in some cases by university funds. In addition, the nonU.S. participants, primarily from Italy, Japan and Russia, who work at the U.S. laboratories receive funding from the science and education ministries of their countries. The majority of U.S. particle physicists carry out their research at Fermilab. Of the approximately 2,200 U.S. physicists actively working in the field today, about 1,300 use Fermilab for their research. Another 400 U.S. researchers work at CERN, on the French-Swiss border, and roughly 50 work at DESY in Germany. The remainder are split about evenly among SLAC, CESR and the AGS.

\section{U.S. Research Facilities}

Two U.S. facilities, the Tevatron and the Cornell Electron Storage Ring, are the premier facilities of their type in the world. The Tevatron is the highest-energy collider in the world and CESR is the highest luminosity collider in the world. The Tevatron defines the energy frontier in particle physics today and will continue to do so for another decade; it provides research opportunities not found anywhere else. The Tevatron's energy gives the experimenters who use the collider detectors, $C D F$ and $D \varnothing$, the unique expectation to discover the top quark and then measure its basic properties once it is found. The Tevatron is also the only accelerator in operation that can produce the force-carrying particles called $\mathrm{W}$ bosons in sufficient quantity to make precise measurements of the W's properties. Within the next few years, scientists working at the Large Electron Positron Collider (LEP) at CERN will acquire a comparable capability of producing the $\mathrm{W}$ boson in large enough numbers to measure its properties.

Measurement of the properties of particles called mesons and baryons, containing $\mathrm{b}$ quarks, has become an important focus of research, because these particles provide clear-cut tests of our theoretical understanding of quarks. Cornell's CESR with the CLEO detector has become the world leader in making the most accurate measurements of many of the properties of the lightest B mesons. In addition, CESR's high luminosity provides the best opportunity to detect and measure the properties of rare but informative decay modes of these B mesons. However, the study of particles containing $b$ quarks is not limited to CESR. For example, measurements made at the Tevatron with the CDF detector, and at CERN's LEP with its four detectors, have provided the best measurements-not possible at CESR - of the lifetime of all of the B mesons and the first evidence for baryons containing $b$ quarks. While all these experiments are pursuing the common goal of understanding the $b$ quark, the ability to measure different properties of $b$ quarks makes them complementary. 
The Stanford Linear Collider (SLC) occupies a unique niche in particle physics, for two reasons: it is the only linear collider in operation, and its electron beam can be polarized. There is consensus in the high-energy physics community that the next step in energy of $\mathrm{e}^{+} \mathrm{e}^{-}$colliders beyond LEP must be made with a linear collider. Because the SLC is serving as the test bed for development of $\mathrm{TeV}$ linear colliders, the work done there is essential for the future. The polarization of the SLC's electron beam allows the most precise measurement of one of the fundamental properties of the $Z^{0}$ particle. The high luminosity of LEP, which also has the capability of studying the $Z^{0}$, is the main attraction for the roughly 400 U.S. experimentalists who choose to work at LEP rather than the SLC. Although the Brookhaven Alternating Gradient Synchrotron is the oldest of operating U.S. accelerators, it still provides a valuable and unduplicated resource for the search for rare decays of $K$ mesons.

There is very little overlap among the research capabilities of the four U.S. accelerator laboratories, although there is some duplication in capability when European laboratories are included. A major strength of the U.S. particle physics program is its diversity, the all-important ability to come at the significant questions of high energy physics in different ways.

Although "measurement of properties" may not sound as exciting or important as "discovery," in fact measurements are the way discoveries are made in particle physics, and indeed in all science. In the 1890s the great British physicist William Thomson, Lord Kelvin, one of the founders of the science of thermodynamics, explained it this way: "When you can measure what you are speaking about, and express it in numbers, you know something about it; but when you cannot measure it, when you cannot express it in numbers, your knowledge is of a meager and unsatisfactory kind: it may be the beginning of knowledge, but you have scarcely, in your thoughts, advanced to the stage of science." To get at the truth and answer our questions in particle physics, we need more than one set of measurements, made by different, complementary means. That is why the diversity of high-energy physics laboratories is so important to the progress of discovery in particle physics.

\section{The Near-Term Requirements of the Base Program}

Over the next decade the U.S. laboratories are capable of providing world-class opportunities for research at the forefront of the field. However, at the present level of funding for high-energy physics research, they will be unable to achieve their potential for excellence. The inadequacy of resources to operate the world-class facilities is a consequence of the very high priority that the field of high-energy physics gave to the construction of the SSC. Funds to operate and upgrade the rest of the high-energy research programcalled the base program - slowly declined over the past five years, as funds for SSC construction rose rapidly. When Congress voted to terminate the 
construction of the SSC, the funding of the base program continued on its downward trajectory. The U.S. high-energy physics community is now left with no SSC in its future and insufficient funding in the present to take good advantage of the opportunities for significant discovery in this decade. The base program needs a modest increase of 10 percent above FY1994 funding, exclusive of funds for construction, if the program is to fulfill its potential for discovery in this decade. Without such an increase, it will be necessary to restructure the base program by eliminating some of its diversity. This will be difficult and damaging in the short term, but it will be necessary for the health of the elements that survive.

To continue among the world leaders in high-energy physics, the U.S. will need some forefront accelerator facilities located here in the United States. Consider for a moment what the U.S. program would be like if it were reduced to using facilities in other regions, with no operating high-energy accelerators in the U.S. The benefits that the U.S. has derived from the development of accelerators since E.O. Lawrence's seminal work on the cyclotron at Berkeley sixty years ago would cease. The U.S. contributions to the field of high-energy physics would dwindle rapidly. The U.S. would not be among world leaders in the field, nor would it be in a position to move into a leadership role, without forefront accelerators in the U.S.

It is important that the U.S. have the capability of building colliders at the energy frontier. If at some time in the distant future the U.S. is to be the site of the highest-energy collider in the world, then we must pursue the development of accelerator technology to bring the cost of such facilities down to an affordable level. The SSC's death has brought the development of superconducting magnet technology, the enabling technology for hadron colliders, to a near standstill in the U.S, and, as a result, we are on the verge of losing our capability in a field where the U.S. has always been the leader.

The modest amounts of construction funds that are provided in the budget are also needed, to build the Main Injector at Fermilab and the B Factory at SLAC. These two projects will enhance the potential of their respective laboratories by strengthening the ability of these existing facilities to provide world-class research opportunities. It is important to complete them rapidly. The B Factory will allow a deeper study of the origin of the matter-antimatter asymmetry than is possible today. The Main Injector will enhance top quark studies and make neutrino beams of unparalleled intensity at an energy level that will allow investigation of the question whether neutrinos have mass. The construction of the Main Injector and the B Factory are necessary if the U.S. is to continue among the world's leaders in the science of high-energy physics. If DOE's plans for the Main Injector and the B Factory are implemented, as scheduled, by 1998, it would then be possible to begin to allocate funds for a new project after 1998. 


\section{The Energy Frontier and the Long-Term Future}

The search for the understanding of the cause of the very different masses of all the known particles remains the central quest in particle physics today. The high-energy community believes that the best path to that understanding is through the construction of a proton-proton collider built with superconducting magnets. The maturity of this technology is amply demonstrated by the Tevatron, the first superconducting collider. The SSC has been terminated, and we know that research at the energy frontier will not proceed by that route in the U.S. for the foreseeable future. The Large Hadron Collider( LHC) proposed by CERN is scheduled for completion early in the next century. While it has only a third of the energy that had been planned for the SSC, it offers the best prospect for U.S. experimentalists to work at the energy frontier in the next decade. U.S. participation in the LHC will probably require a contribution of roughly $\$ 300$ million to CERN for the construction of LHC and another $\$ 200$ million for the U.S. contribution to the construction of the two detectors. This would require a 10 percent increase in high-energy physics funding, in addition to funding for the base program, over the ten-year period between 1995 and 2005-a very small number compared to the funds previously allocated to the SSC. This is the only affordable option open to the U.S. high-energy community if it hopes to continue to work at the energy frontier. The issues of the desirability of U.S. participation in the LHC program, as well as the proper balance between the research opportunities in this decade and those of the next, will be addressed by the recently appointed HEPAP subpanel on the future of high-energy physics.

\section{Beyond Accelerators}

Particle physics research advances not only with accelerators but by the study of the early universe. In recent years, the convergence of particle physics, cosmology, and astrophysics has grown increasingly clear. The interplay of these different fields promises to continue into the next century. Discoveries at accelerators will have significant impact on astrophysics and cosmic-ray physics; and progress in those fields will in turn illuminate our understanding of the structure of matter. For some time, large underground experiments designed to observe the interaction of neutrinos from outer space and large counter arrays at the earth's surface, designed to detect highenergy cosmic rays, have been underway. Their observations have raised questions that may have very far-reaching answers. Fermilab has recently joined with astrophysicists to play an important part in fostering this convergence, through its part in the Sloan Digital Sky Survey, which will provide information about large-scale structure of the universe.

\section{A Tradition of Consensus-the HEPAP Process}

The high-energy physics community has a strong tradition of reaching formal consensus within the field on the best use of scarce resources for particle physics research. For years, HEPAP, the High Energy Physics 
Advisory Panel to DOE, has made detailed determinations of research priorities within the constraints of federal budgets. For some questions, a subpanel is established to study the issue in depth. Since its establishment in 1967, HEPAP has issued 36 subpanel reports on topics ranging from technical issues to broad studies of the future direction of particle physics in general. Although HEPAP operates under the auspices of DOE, its reports address the whole U.S. particle physics program, including the portions supported by NSF. A subpanel of HEPAP is now at work in the effort to forge a consensus on direction and priorities for future U.S. research in the absence of the SSC.

\section{Conclusion}

The termination of the SSC has created a crisis for the U.S. high-energy program. The funding for the base program is no longer sufficient to allow achievement of the great potential for discovery in the U.S., and it certainly does not allow for new projects. Nevertheless, some superb opportunities exist, and they can be realized with a modest increase in funding. Without an increase, the field will need to be restructured to ensure the health of the surviving elements. If this is the direction we must take, a process such as HEPAP's is the proper way to chart the course. 